\section{Hyperthermia: Different Methods, Different Aims, and Different Problems}

\section{Concerning Feyerabend T., Wiedemann G.J., Richter E., Hegewisch-Becker S.: Hyperthermia as an adjunct to the standard treatment in neo- plastic diseases: Few cures but some advances. Onkologie 1999;22:122-127.}

With great interest we read the article by Feyerabend et al. focussing on the potential of different methods of hyperthermia in the treatment of malignant diseases. We are also involved in the clinical evaluation of different forms of hyperthermia and, therefore, we would like to add a few points to the above-mentioned presentation.

\section{Molecular and Immunological Effects}

In our view, the introduction of the article leads to the impression that preclinical studies on hyperthermia have already resulted in profound understanding of the molecular basics of hyperthermia. The connections, however, between apoptosis and thermoresistency (not mentioned by the authors) in an organism subjected to elevated temperatures raise a continuum of questions, for example on details of the immunological processes, which can be caused by hyperthermia and which to some extent are mediated by heat shock proteins. In this context, to us the mere phenomenological changes of the white blood cells and of lymphocyte subpopulations observed during hyperthermia may be interpreted too simply. From our own experience we know that it is extremely difficult to extract relevant and independent aspects of the molecular and immunological effects of hyperthermia from investigations which are part of clinical studies, as - among other reasons - the application of hyperthermia, usually, is only part of schemes of treatment involving several different therapeutical modalities. On the one hand, therefore, by our own investigations we can confirm that complex immunological changes like the increase of NK cells in peripheral blood, also mentioned by Feyerabend et al., as well as changes of concentrations of different soluble adhesion molecules and of cytokines take place in patients having undergone whole-body hyperthermia. On the other hand, it is not yet clear to what extend these phenomena differ significantly from those changes which are observed in other stress situations (e.g. polytrauma), thereby reducing the possible significance of these changes for the explanation of the effect of hyperthermia.

\section{Modalities of Hyperthermia}

In our view, optimism regarding the application of the different modalities of hyperthermia, besides persuading results obtained from in vitro models, is mainly due to encouraging clinical results. In this context, one should, however, differentiate more clearly between the different modalities of hyperthermia. The clinical benefits of local and regional hyperthermia combined with radiotherapy and/or cytostatic treatment are, nowadays, probably the best verified, although, so far, this strategy of treatment has not become a generally recommended, standardized form of treatment. Above that, interstitial hyperthermia, hyperthermic isolated perfusion of extremities (HIE), and whole-body hyperthermia $(\mathrm{WBH})$ induced by radiation procedures with acceptable toxicity have achieved promising results. These methods have different therapeutical potentials: Regional, local, and interstitial hyperthermia do have the problem of inducing homogenous intratumoral temperatures, whereas HIE and WBH achieve homogenous temperature distribution within the treated region. Local and regional hyperthermia may be employed for controlling the growth of locally progressing neoplasms, whereas whole-body hyperthermia represents a systemic form of therapy for overt or possibly disseminated malignomas or hematological diseases.

Due to this variety of the different modalities of hyperthermia, which may seem confusing to the non-expert, a widely accepted, exact terminology is desirable. Unfortunately, the expressions 'hyperthermia' and 'local/regional hyperthermia' were partly used synonymously in the article on the treatment of locally progressive tumours by Feyerabend et al., and we are afraid that in some parts of the paper there is a lack of differentiation between local and regional hyperthermal procedures, although both are procedures demanding greatly differing expenditure of apparatus and logistics. Although local or regional hyperthermia has not yet been accepted as a standard form of therapy for some indications, its effectiveness in some indications has been confirmed in valid phase-III-studies (e.g. Overgaard et al., Lancet 1995;345:540ff.). Promising results on the effectiveness of hyperthermia have also been delivered by a few well-published phase II studies, which evaluate combinations of regional hyperthermia, radiation, and cytostatic therapy. Especially the study by Issels et al. (JCO 1990;8: 1818ff.) gave encouraging results with regard to neoadjuvant therapy of soft tissue sarcomas and has therefore been continued as a still running phase III study of the EORTC. One of the major results of this study was the correlation of the thermal dose, applied to the neoplastic tissue, with the degree of the clinical benefit. These findings correspond with our experiences with preoperative radiochemotherapy plus regional hyperthermia in locally advanced carcinomas of the rectum (Rau et al., Ann Surg 1998;227:380ff.). Indeed, we would have preferred a more detailed discussion of the different aspects of regional hyperthermia in upcoming concepts of neoadjuvant therapy.

\section{Whole-Body Hyperthermia}

Regarding the discussion of whole-body hyperthermia we would, firstly, like to remark, that an applicator which uses infrared radiation as temperature-increasing principle and which is comparable to an Aquatherm ${ }^{\mathrm{TM}}$, was already applied

\begin{tabular}{ll}
\hline KARGER & $\oplus 1999$ S. Karger GmbH, Freiburg \\
$\begin{array}{l}\text { Fax }+497614520714 \\
\begin{array}{l}\text { E-mail kargergmbh@aol.com } \\
\text { www.karger.com }\end{array}\end{array}$ & Accessible online at: \\
& http://BioMedNet.com/karger
\end{tabular}


in a phase I study by Robins in 1985 and not only in 1993 (Cancer Res 1985;45:3937-3944). Unfortunately, another advanced system for the induction of whole-body hyperthermia in clinical practice, the Iratherm ${ }^{\circledR} 2000$, developed by the 'von Ardenne Institute' in Dresden, was not mentioned. We consider this applicator to be an alternative to the Aquatherm, as both systems do not differ to a great extent regarding the spectrum of toxicity and potential effectiveness. In this context, we also believe that the differentiation between the concepts of whole-body hyperthermia using the Aquatherm device, and von Ardenne's 'systemic Cancer Multistep Therapy' (sCMT) using the Iratherm-2000 apparatus, is only of marginal importance, as high blood glucose level $(300-400 \mathrm{mg} / \mathrm{dl})$ as well as hyperoxaemia are induced in both settings.

Undoubtedly, we would have asked for a detailed differentiation between these two applicators and agree, however, that the principle of extracorporally inducing whole-body hyper- thermia, which was discussed by Feyerabend et al. in detail, is associated with a high risk of severe to most severe toxicity and that its application should therefore be stopped. On the other side, it remains unclear to what extent the radiant heat devices Iratherm-2000 and Aquatherm are competitive in clinical practice, and thus we think that a direct comparison of both machines is necessary in the future in order to find out possible differences with regard to the safety of treatment and therapyassociated toxicity.

\section{B. Hildebrandt, J. Loeffel, O. Ahlers ${ }^{\mathrm{a}}$, P. Wust ${ }^{\mathrm{a}}$, H. Riess}

Medizinische Klinik für Hämatologie und Onkologie und a Strahlenklinik, Charité, Medizinische Fakultät der Humboldt Universität, Campus Virchow Klinikum

Augustenburger Platz, D-13353 Berlin (Germany), Tel. +49 30 450-53112, Fax -53901 